\title{
What is the most sensitive non-invasive imaging strategy for the diagnosis of intracranial aneurysms?
}

\author{
P M White, E Teadsale, J M Wardlaw, V Easton
}

\begin{abstract}
Objectives-To determine whether combining non-invasive tests for intracranial aneurysms together would significantly improve aneurysm detection over individual tests.

Methods-114 patients undergoing intraarterial digital subtraction angiography to confirm or exclude an intracranial aneurysm were also examined by CT angiography, $M R$ angiography, and transcranial power Doppler ultrasound. The reviewers and ultrasonographers were blinded to the angiogram result, other imaging results and all clinical information.
\end{abstract}

Results-The combination of noninvasive tests did improve diagnostic performance on a per patient basis. The combination of power Doppler and CT angiography had the greatest sensitivity for aneurysm detection $(0.83 ; 05 \%$ confidence interval (95\% CI) $0.66-0.93)$ and the level of agreement for this strategy with the reference angiographic standard was excellent (к $0.84 ; 95 \%$ CI 0.72-0.95). The improvement in sensitivity of adding power Doppler to CT angiography was not significant $(p=0.55)$ but the improvement in the level of agreement with the reference standard was substantial. However, even the most sensitive combination strategy performed poorly in the detection of small $(3-5 \mathrm{~mm})$ and very small $(<3 \mathrm{~mm})$ aneurysms with a sensitivity of $0.43(95 \%$ CI 0.23-0.66) and 0.00 (95\% CI 0.00-0.31) respectively.

Conclusions-The addition of transcranial power Doppler ultrasound to either CT angiography or MR angiography does improve diagnostic performance on a per patient basis but aneurysms of $5 \mathrm{~mm}$ or smaller can still not be reliably identified by current standard clinical non-invasive imaging modalities.

(F Neurol Neurosurg Psychiatry 2001;71:322-328)

Keywords: intracranial aneurysms; CT angiography; MR angiography; transcranial power Doppler

University of

Edinburgh

Department of

Medical Statistics,

Medical School, Teviot

Place, Edinburgh, UK

V Easton

Correspondence to:

Dr PM White

pmw@skull.dcn.ed.ac.uk

Received 10 October 2000 and in revised form

26 February 2001

Accepted 7 March 2001 have compared non-invasive techniques such as MR angiography, CT angiography, or transcranial Doppler ultrasound (TCD) to IADSA. ${ }^{6}$ These have found similar overall accuracy/ aneurysm for CTA and MRA of about $90 \%$ but with sensitivity ranging from 0.67 (95\%CI $0.55-0.78)^{7}$ to $1.0(95 \% \text { CI } 0.85-1)^{8}$ for CTA and $0.70(95 \% \mathrm{CI} 0.5-0.86)^{9}$ to $0.97(95 \% \mathrm{CI}$ $0.87-1)^{10}$ for MRA. The data on TCD are much more limited but overall accuracy/aneurysm appears lower, in the range $0.57^{11}-0.80 .^{5}$ The main drawback of all the non-invasive tests has been their low sensitivity compared with IADSA, particularly for small aneurysms (those $\leqslant 5 \mathrm{~mm}$ in maximum diameter).

Despite its possible limitations as an isolated investigation, TCD is an attractive technique in the investigation of intracranial aneurysms particularly in "screening" for unruptured aneurysms because of its safety, rapidity, repeatability, mobility, and lower cost compared with the other techniques. ${ }^{11}$ Our aim, therefore, was to determine, in a fully blinded prospective study, whether a strategy utilising a combination of power TCD and either CTA or MRA, or CTA and MRA, could improve the sensitivity of a non-invasive investigation strategy for intracranial aneurysms. We also wished to determine which tests were preferred by patients.

Subjects and methods

Approval for the study was obtained from the appropriate hospital ethics committees and written informed consent was obtained from participants.

\section{PATIENTS}

The study was conducted in two regional neuroscience centres serving a population of 4.2 million. Patients undergoing cerebral angiography for the detection of a possible intracranial aneurysm were eligible for inclusion. Exclusion criteria were patients with a poor grade of subarachnoid haemorrhage (World Federation of Neurosurgeons grade 3 or worse) because obtaining informed consent was not possible, patients with an absolute contraindication to one of the examinations, or patients less than 18 or older than 75 years of age. Two hundred consecutive patients meeting the inclusion criteria and who agreed to participate were recruited prospectively over an 18 month period, of whom 173 underwent CTA, 152 MRA, and 171 TCD as well as IADSA examination. One hundred and thirty $(65 \%)$ patients underwent all three non-invasive tests but of these, five were excluded because they were unable to complete the MRA examination and a further 11 patients had an inadequate bone window on TCD so were also excluded, resulting in our study population of 114 patients ( 55 men and 59 women); 
median age 41 (range 19-71 years). Imaging studies were performed contemporaneously (within a week of IADSA) if at all possible, and within a maximum of 2 months from the time of IADSA; $54 \%$ of CTA, $45 \%$ of MRA, and $61 \%$ of TCD examinations were performed contemporaneously, with a further $6 \%, 4 \%$, and $8 \%$ respectively being performed within 2 weeks of IADSA.

Patients were grouped into four categories based on the clinical indication for cerebral angiography. Group 1 comprised three patients with a known aneurysm(s) undergoing further assessment; group 2, 44 patients with proved subarachnoid haemorrhage; group 3, 52 patients with symptoms which might be due to an aneurysm, and group 4, 15 asymptomatic patients at risk of harbouring an aneurysm.

IMAGE ACQUISITION

Neuroradiologists performed all IADSA examinations using GE Advantx angiographic equipment (IGE Ltd, Milawaukee, USA). The IADSA studies were three or four vessel selective angiograms with multiple projections obtained for each vessel.

\section{CTA TECHNIQUE}

Spiral CTA examinations were performed on Elscint Twin or GE HiSpeed machines using a standard technique. ${ }^{3} 100 \mathrm{ml}$ non-ionic contrast were given by pump injector into an antecubital vein at $3 \mathrm{ml} / \mathrm{s}$ with an $18-20$ s delay. Examination protocol was $120 \mathrm{kV}$, maximum tube current allowed, $512 \times 512$ matrix, $15 \mathrm{~cm}$ FOV, $1 \mathrm{~mm}$ collimation, and pitch of 1.5 with 0.5 $\mathrm{mm}$ reconstruction interval. For logistical reasons, a few patients (12) had a non-spiral CTA performed on an Elscint 2400 Elite scanner. Conventional axial CTA was performed dynamically using $2.5 \mathrm{~mm}$ slice width with 1 $\mathrm{mm}$ table increment, $120 \mathrm{kV}, 400 \mathrm{mAs}, 20 \mathrm{~cm}$ FOV. Scanning started after $50 \mathrm{ml}$ contrast had been injected rapidly by hand with a further 50 $\mathrm{ml}$ injected during scanning. ${ }^{1}$

MAGNETIC RESONANCE ANGIOGRAPHY TECHNIQUE Magnetic resonance angiography examinations were performed on Elscint Prestige 2T or Siemens Magnetom SP 1.5T machines using 3D time of flight (TOF) MRA sequences with MTS and TONE followed by a T2 FSE axial sequence using a standard technique. On the Prestige, settings were TR $40 \mathrm{~ms}$, TE $6 \mathrm{~ms}$, flip angle 300 , FOV $15 \times 20 \mathrm{~cm}, 204 \times 300$ matrix, NEX 1, TA=7:53. On the Magnetom, settings were TR $43 \mathrm{~ms}$, TE $8 \mathrm{~ms}$, flip angle 200 , FOV $20 \mathrm{~cm}, 256 / 512$ / oversampled matrix, $\mathrm{NEX}=1$, $\mathrm{TA}=1$ 1.48. Neuroradiologists supervised all CTA and MRA examinations.

ULTRASOUND TECHNIQUE

Transcranial power Doppler ultrasound examinations were performed on Acuson 128XP machines using 2-2.5 MHz multihertz linear transducers (Acuson, Mountain View, California, USA). Identical imaging settings were used on the machine in each centre. Transcranial Doppler examinations were performed via the temporal bone window to insonate the circle of
Willis in the axial and coronal planes. ${ }^{12-15}$ The transnuchal and transorbital routes were not routinely employed and intravenous echo contrast was not used. Each major intracranial vessel segment was examined systematically using power and spectral Doppler ultrasound. Aneurysm size was determined on a frozen image using electronic calipers. A video record of each examination was made and a standard result proforma sheet was completed at the end of each examination. Ultrasonographers comprising two neuroradiologists and three neuroradiographers (two very experienced in power transcranial Doppler and three less experienced), were blinded to clinical data and the results of all other imaging investigations including plain CT and IADSA results.

\section{POSTPROCESSING OF IMAGES}

For CTA studies, reformatting of source images was performed by a neuroradiology research fellow on offline workstations without review of the IADSA study before performing reformats (Silicon Graphics O2 Omnipro or GE Advantage Windows). Standard axial, coronal oblique, and curved sagittal multiplanar reformats were performed on the Omnipro. " "Angio MIP" (maximum intensity projection) reconstructions were also performed (12 projections at $15^{\circ}$ intervals in both "head over heels" and "left to right" projections), with bone editing by thresholding and manual cutting. Targeted MIPs were performed of the right and left internal carotid circulations and the vertebrobasilar system. The total time taken for these reconstructions was typically 20-25 minutes. On the advantage windows workstation, axial, coronal oblique, and sagittal overlapping thick slab MIP images $(8-10 \mathrm{~mm}$ at $3-4 \mathrm{~mm}$ increments) were performed, with additional manual bone editing as required. ${ }^{2}$ Reconstruction time was less than 10 minutes unless manual bone editing was required. For MRA, standard MIP reconstructions were performed at $15^{\circ}$ intervals through $180^{\circ}$ (in "head over heels" and "left to right" projections) at the time of examination by the neuroradiographer performing the examination. Targeted MIP reconstructions were performed for examinations performed on the Prestige machines on the Omnipro workstation, as in the CTA technique. Source images were available to reviewers for CTA and MRA examinations.

\section{IMAGE REVIEW}

The IADSA images were presented on hard copy as anonymised, randomly numbered studies with no clinical details or results of other imaging for independent review by two consultant neuroradiologists (JMW, ET). Where disagreements arose these were resolved by consensus review. For TCD, the report completed at the end of each examination was used for the comparison with IADSA. The CTA and MRA studies were presented in an anonymised, random fashion to the same two neuroradiologists, they were reviewed separately and at least 4 months elapsed between review of an angiogram and reviewing the noninvasive studies on the same patient. For the 
Table 1 Diagnostic performance per patient for different imaging strategies

\begin{tabular}{lll}
\hline Strategy & Sensitivity $(95 \%$ CI $)(T P / T P+F N)$ & Specificity $(95 \%$ CI $)(T N / T N+F P)$ \\
\hline CTA $^{\star}$ & $0.80(0.65-0.90)(36 / 45)$ & $0.91(0.82-0.97)(63 / 69)$ \\
MRA $^{\star}$ & $0.71(0.56-0.84)(32 / 45)$ & $0.97(0.90-1.00)(67 / 69)$ \\
TCD & $0.73(0.58-0.85)(33 / 45)$ & $0.91(0.82-0.97)(63 / 69)$ \\
CTA+TCD & $0.83(0.66-0.93)(29 / 35)$ & $0.98(0.91-1.00)(58 / 59)$ \\
MRA+TCD & $0.76(0.59-0.88)(28 / 37)$ & $1.00(0.94-1.00)(61 / 61)$ \\
CTA+MRA & $0.79(0.64-0.91)(31 / 39)$ & $1.00(0.94-1.00)(61 / 61)$
\end{tabular}

^Results used for CTA and MRA were those of the "better" observer, although the results for both observers were very similar (see note in discussion). For the "poorer" observer, sensitivity per patient (PP) was 0.82 and 0.71 for CTA and MRA respectively. For combination strategies, sensitivity PP was $0.86,0.79$, and 0.85 for CTA+TCD, MRA+TCD, and CTA+MRA respectively. Where tests disagreed, the result was classified as "uncertain", necessitating confirmatory IADSA rather than being classified as a true positive (TP), true negative (TN), false positive (FP), or false negative $(\mathrm{FN})$ result. For CTA+TCD there were 20 "uncertain" cases, 16 for MRA+TCD and 14 for CTA+MRA.

Table 2 Diagnostic performance per aneurysm for non-invasive imaging tests

\begin{tabular}{llll}
\hline Modality & $\begin{array}{l}\text { Size of } \\
\text { aneurysm }\end{array}$ & $\begin{array}{l}\text { Sensitivity }(95 \% \mathrm{CI}) \\
(T P / T P+F N)\end{array}$ & $\begin{array}{l}\text { Specificity }(95 \% \mathrm{CI}) \\
(T N / T N+F P)\end{array}$ \\
\hline CTA & $<3 \mathrm{~mm}$ & $0.40(0.19-0.64)(8 / 20)$ & $0.91(0.82-0.97)(63 / 69)$ \\
& $3-5 \mathrm{~mm}$ & $0.56(0.40-0.72)(22 / 39)$ & $0.88(0.78-0.94)(63 / 72)$ \\
& $5.1-10 \mathrm{~mm}$ & $0.83(0.52-0.98)(10 / 12)$ & $0.98(0.91-1.00)(63 / 64)$ \\
& $>10 \mathrm{~mm}$ & $1.00(0.72-1.00)(11 / 11)$ & $1.00(0.94-1.00)(63 / 63)$ \\
& All sizes & $0.62(0.51-0.73)(51 / 82)$ & $0.80(0.69-0.88)(63 / 77)$ \\
MRA & $<3 \mathrm{~mm}$ & $0.15(0.03-0.38)(3 / 20)$ & $0.93(0.85-0.98)(67 / 72)$ \\
& $3-5 \mathrm{~mm}$ & $0.38(0.23-0.55)(15 / 39)$ & $0.99(0.92-1.00)(67 / 68)$ \\
& $5.1-10 \mathrm{~mm}$ & $0.75(0.43-0.95)(9 / 12)$ & $1.00(0.95-1.00)(67 / 67)$ \\
& $>10 \mathrm{~mm}$ & $0.91(0.59-1.00)(10 / 11)$ & $1.00(0.95-1.00)(67 / 67)$ \\
TCD & All sizes & $0.45(0.34-0.57)(37 / 82)$ & $0.92(0.83-0.97)(67 / 73)$ \\
& $<3 \mathrm{~mm}$ & $0.15(0.03-0.38)(3 / 20)$ & - \\
& $3-5 \mathrm{~mm}$ & $0.36(0.21-0.53)(14 / 39)$ & - \\
& $5.1-10 \mathrm{~mm}$ & $0.42(0.15-0.72)(5 / 12)$ & - \\
& $>10 \mathrm{~mm}$ & $0.64(0.31-0.89)(7 / 11)$ & - \\
& All sizes & $0.35(0.25-0.47)(29 / 82)$ & $0.73(0.63-0.82)(63 / 86)$
\end{tabular}

^For TCD, size categorisation was not available for all the false positive cases; therefore, specificity and accuracy could not be determined by aneurysm size category.

purpose of subgroup analysis, aneurysms were grouped into four size categories: $(1)<3 \mathrm{~mm}$ maximum angiographic dimension, (2) 3-5 $\mathrm{mm}$, (3) $5.1-10 \mathrm{~mm}$, and (4) $>10 \mathrm{~mm}$.

PATIENT FEEDBACK

All participants were sent a questionnaire 1-2 weeks after completing the study examinations. This asked them to grade the discomfort experienced during each test on a $10 \mathrm{~cm}$ long visual analogue scale $(0$ being no discomfort and 10 being as uncomfortable or painful as they have ever experienced) and to rank the three non-invasive tests in order of preference. A prepaid and addressed envelope was provided with the questionnaire.

\section{STATISTICAL METHODS}

$2 \times 2$ tables were constructed of true positives, false positives, false negatives, and true negatives for each modality compared with the gold standard (IADSA) on a per patient and per aneurysm basis. Sensitivity, specificity, positive and negative predictive values, and accuracy were calculated and compared on a per patient and a per aneurysm basis for the following investigative strategies: (a) CTA alone, (b) MRA alone, (c) TCD alone, (d) CTA+TCD, (e) MRA+TCD, and ( $f$ ) CTA+MRA. Per patient basis means the ability to correctly discriminate a patient as true positive or true negative for possession of at least one intracranial aneurysm and per aneurysm basis the ability to correctly identify all aneurysms. Exact $95 \%$ confidence intervals (95\% CIs) based on binomial probabilities were calculated. ${ }^{16}$ Unweighted $\kappa$ statistic was used to assess the level of intermodality and interobserver agreement. ${ }^{17} \mathrm{~A} \kappa$ value of $\leqslant 0.20$ implies poor agreement, of 0.21-0.40 fair agreement, of 0.41-0.60 moderate agreement, of $0.61-0.80$ good agreement, and of $0.81-1.0$ very good agreement. ${ }^{17}$

\section{Results}

Three patients experienced minor complications from IADSA (one groin haematoma and two nausea and vomiting); one had a moderate delayed contrast reaction after CTA (that responded rapidly to oral antihistamine and steroid); two patients experienced claustrophobia during MRA but were able to complete the examination and five patients were unable to tolerate the MRA examination; one patient reported mild scalp discomfort from the TCD probe.

The diagnostic performance of both observers (for CTA and MRA) was similar and there was good interobserver agreement for CTA and MRA, with $\kappa$ values of 0.69 (95\% CI $0.56-$ $0.83)$ and $0.73(95 \%$ CI $0.60-0.87)$ respectively. Overall, observer B had slightly better results with an accuracy per patient of 0.87 (95\% CI 0.79-0.92) for both CTA and MRA compared with $0.81(95 \%$ CI $0.72-0.87)$ for CTA and 0.84 (95\% CI 0.76-0.90) for MRA for observer $\mathrm{A}$. The results of observer $\mathrm{B}$ were used to evaluate further the different possible non-invasive imaging strategies.

The sensitivity and specificity of the different imaging strategies are given in full on a per patient basis in table 1 . The sensitivity for CTA+TCD was 0.83 (95\% CI 0.66-0.93); for MRA+TCD it was 0.76 (95\% CI 0.59-0.88) and for CTA+MRA it was 0.79 (95\% CI 0.640.91). Table 2 relates the performance of the non-invasive tests on a per aneurysm basis to the aneurysm size and shows that CTA and MRA performed substantially better than TCD for larger aneurysms and that each noninvasive method performed much worse in the detection of small aneurysms. Table 3 indicates the effect on sensitivity on a per aneurysm basis from combining non-invasive tests together. Sensitivity was reduced on a per aneurysm basis by combining tests together, the methods frequently disagreed on the presence of an individual aneurysm or on the precise location and size of aneurysm (as indicated by the number of cases falling into the "uncertain" category).

Despite the trend demonstrated for a combination of tests to improve the sensitivity and overall accuracy of non-invasive imaging on a per patient basis, the extent of improvement did not reach significance. The statistical parameters for the increase in sensitivity of adding TCD to CTA, TCD to MRA, and MRA to CTA were respectively $\mathrm{p}=0.55\left(\chi^{2}\right.$ $0.37), 0.50\left(\chi^{2} 0.46\right)$, and $0.95\left(\chi^{2} 0.004\right)$ and for the improvement in accuracy were $0.16\left(\chi^{2}\right.$ 1.95), 0.18 ( $\left.\chi^{2} 1.75\right)$, and 0.18 ( $\chi 2$ 1.75).

The level of agreement for each method with the reference standard and with the other noninvasive tests was determined using the $\kappa$ value (with 95\% CI calculated in all cases). This method was also used to determine the level of 
Table 3 Sensitivity per aneurysm for combinations of non-invasive tests

\begin{tabular}{|c|c|c|c|}
\hline Modality & $\begin{array}{l}\text { Size of } \\
\text { aneurysm }\end{array}$ & $\begin{array}{l}\text { Sensitivity (95\% CI) } \\
(T P / T P+F N)\end{array}$ & $\begin{array}{l}\text { Specificity }(95 \% \text { CI) } \\
(T N / T N+F P)\end{array}$ \\
\hline $\begin{array}{l}\text { CTA+TCD* } \\
\text { (64 "uncertain") }\end{array}$ & $\begin{array}{l}<3 \mathrm{~mm} \\
3-5 \mathrm{~mm} \\
5.1-10 \mathrm{~mm} \\
>10 \mathrm{~mm} \\
\text { All }\end{array}$ & $\begin{array}{l}0.00(0.00-0.31)(0 / 10) \\
0.43(0.23-0.66)(10 / 23) \\
0.71(0.29-0.96)(5 / 7) \\
1.00(0.59-1.00)(7 / 7) \\
0.47(0.32-0.62)(22 / 47)\end{array}$ & $\begin{array}{l}- \\
- \\
\overline{-} \\
0.95(0.86-0.99)(58 / 61)\end{array}$ \\
\hline $\begin{array}{l}\text { MRA+TCD` } \\
\text { (55 “uncertain") }\end{array}$ & $\begin{array}{l}<3 \mathrm{~mm} \\
3-5 \mathrm{~mm} \\
5.1-10 \mathrm{~mm} \\
>10 \mathrm{~mm} \\
\text { All }\end{array}$ & $\begin{array}{l}0.00(0.00-0.22)(0 / 15) \\
0.26(0.10-0.48)(6 / 23) \\
0.63(0.24-0.91)(5 / 8) \\
1.00(0.54-1.00)(10 / 10) \\
0.38(0.25-0.51)(21 / 56)\end{array}$ & $\begin{array}{l}- \\
- \\
\overline{-} \\
0.97(0.89-1.00)(61 / 63)\end{array}$ \\
\hline $\begin{array}{l}\text { CTA+MRA } \\
\text { (34 "uncertain") }\end{array}$ & $\begin{array}{l}<3 \mathrm{~mm} \\
3-5 \mathrm{~mm} \\
5.1-10 \mathrm{~mm} \\
>10 \mathrm{~mm} \\
\text { All }\end{array}$ & $\begin{array}{l}0.20(0.04-0.48)(3 / 15) \\
0.50(0.31-0.69)(15 / 30) \\
0.89(0.52-1.00)(8 / 9) \\
1.00(0.69-1.00)(10 / 10) \\
0.56(0.43-0.69)(36 / 64)\end{array}$ & $\begin{array}{l}0.98(0.91-1.00)(61 / 62) \\
1.00(0.94-1.00)(61 / 61) \\
1.00(0.94-1.00)(61 / 61) \\
1.00(0.94-1.00)(61 / 61) \\
0.98(0.91-1.00)(61 / 62)\end{array}$ \\
\hline
\end{tabular}

"Uncertain" indicates that the tests disagreed about the presence of an aneurysm.

^For TCD, size categorisation was not available for all the false positive cases, therefore specificity and accuracy could not be determined by aneurysm size category.

Table 4 Level of agreement between modalities (singly and in combination) and the reference standard and with each other determined using unweighted $\kappa$ statistic

\begin{tabular}{ll}
\hline Modalities compared & $\kappa(95 \%$ CI $)$ \\
\hline CTA with IADSA per patient (PP) & $0.72(0.59-0.85)$ \\
MRA with IADSA PP & $0.71(0.58-0.85)$ \\
TCD with IADSA PP & $0.66(0.52-0.81)$ \\
CTA with MRA PP & $0.73(0.59-0.86)$ \\
CTA with TCD PP & $0.60(0.45-0.75)$ \\
MRA with TCD PP & $0.66(0.51-0.81)$ \\
CTA with MRA per aneurysm & $0.69(0.56-0.81)$ \\
CTA with TCD per aneurysm & $0.44(0.27-0.61)$ \\
MRAwith TCD per aneurysm & $0.50(0.32-0.68)$ \\
CTA+TCD with IADSA PP & $0.84(0.72-0.95)$ \\
MRA+TCD with IADSA PP & $0.79(0.69-0.94)$ \\
CTA+MRA with IADSA PP & $0.83(0.71-0.94)$ \\
\hline
\end{tabular}

$\kappa \leqslant 0.20$ indicates poor agreement, of $0.21-0.40$ fair agreement, of $0.41-0.60$ moderate agreement, of $0.61-0.80$ good agreement, and of $>0.80$ very good agreement.

*These results apply where the non-invasive tests agreed with each other. Where they disagreed the result was classified as "uncertain" and not included in the calculation of $\kappa$.

agreement for the combinations of noninvasive tests with IADSA. These results are summarised in table 4 . The agreement between non-invasive modalities and IADSA on a per patient basis (PP) was good. The agreement of the non-invasive tests with each other

Table 5 Sensitivity for aneurysm detection per patient according to maximum aneurysmal size

\begin{tabular}{|c|c|c|}
\hline Modality & $\begin{array}{l}\text { Size of subjects' largest } \\
\text { aneurysm }\end{array}$ & $\begin{array}{l}\text { Sensitivity) }(95 \% C I) \\
(T P / T P+F N)\end{array}$ \\
\hline CTA & $\begin{array}{l}<3 \mathrm{~mm} \\
3-5 \mathrm{~mm} \\
>5.1-10 \mathrm{~mm} \\
>10 \mathrm{~mm}\end{array}$ & $\begin{array}{l}0.25(0.03-0.65)(2 / 8) \\
0.82(0.57-0.96)(14 / 17) \\
0.89(0.52-1.00)(8 / 9) \\
1.00(0.72-1.00)(11 / 11)\end{array}$ \\
\hline MRA & $\begin{array}{l}<3 \mathrm{~mm} \\
3-5 \mathrm{~mm} \\
5.1-10 \mathrm{~mm} \\
>10 \mathrm{~mm}\end{array}$ & $\begin{array}{l}0.125(0.00-0.53)(1 / 8) \\
0.71(0.44-0.90)(12 / 17) \\
0.89(0.52-1.00)(8 / 9) \\
1.00(0.72-1.00)(11 / 11)\end{array}$ \\
\hline $\mathrm{TCD}^{\star}$ & $\begin{array}{l}<3 \mathrm{~mm} \\
3-5 \mathrm{~mm} \\
5.1-10 \mathrm{~mm} \\
>10 \mathrm{~mm}\end{array}$ & $\begin{array}{l}0.25(0.03-0.65)(2 / 8) \\
0.59(0.33-0.82)(10 / 17) \\
0.56(0.21-0.86)(5 / 9) \\
0.82(0.48-0.98)(9 / 11)\end{array}$ \\
\hline $\begin{array}{l}\text { CTA+TCD } \\
(20 / 114 \text { "uncertain" and } \\
10 / 20 \text { had aneurysm on IADSA) }\end{array}$ & $\begin{array}{l}<3 \mathrm{~mm} \\
3-5 \mathrm{~mm} \\
5.1-10 \mathrm{~mm} \\
>10 \mathrm{~mm}\end{array}$ & $\begin{array}{l}0.17(0.00-0.64)(1 / 6) \\
0.92(0.62-1.00)(11 / 12) \\
1.00(0.59-1.00)(7 / 7) \\
1.00(0.69-1.00)(10 / 10)\end{array}$ \\
\hline $\begin{array}{l}\text { MRA+TCD } \\
\text { (16/114 "uncertain" and } \\
8 / 16 \text { had aneurysm on IADSA) }\end{array}$ & $\begin{array}{l}<3 \mathrm{~mm} \\
3-5 \mathrm{~mm} \\
5.1-10 \mathrm{~mm} \\
>10 \mathrm{~mm}\end{array}$ & $\begin{array}{l}0.14(0.00-0.58)(1 / 7) \\
0.75(0.43-0.95)(9 / 12) \\
1.00(0.63-1.00)(8 / 8) \\
1.00(0.69-1.00)(10 / 10)\end{array}$ \\
\hline $\begin{array}{l}\text { CTA+MRA } \\
\text { (14/114 "uncertain" and } \\
6 / 14 \text { had aneurysm on IADSA) }\end{array}$ & $\begin{array}{l}<3 \mathrm{~mm} \\
3-5 \mathrm{~mm} \\
5.1-10 \mathrm{~mm} \\
>10 \mathrm{~mm}\end{array}$ & $\begin{array}{l}0.14(0.00-0.58)(1 / 7) \\
0.85(0.55-0.98)(11 / 13) \\
1.00(0.63-1.00)(8 / 8) \\
1.00(0.72-1.0)(11 / 11)\end{array}$ \\
\hline
\end{tabular}

was also good except for CTA and TCD where agreement was only moderate. In comparison, agreement between the modalities was poorer on a per aneurysm basis, particularly between TCD and either CTA or MRA. The improvement in $\kappa$ was substantial for all combination strategies with an improvement to a "very good" level of agreement with the reference standard for the CTA+TCD and CTA+MRA strategies.

In table 5 , sensitivity results are again given on a per patient basis but stratified according to the largest sized aneurysm that each patient had (whether or not the non-invasive test(s) detected it). The results on a per patient basis for the combination strategies were excellent for aneurysms larger than $5 \mathrm{~mm}$ in maximum angiographic diameter with a sensitivity, specificity, and accuracy of 1.0 for all three combination strategies. Of course a certain number of cases where the non-invasive tests disagreed were classed as "uncertain" and were therefore excluded from these analyses-on the basis that if this disagreement occurred in clinical practice, confirmatory IADSA would be mandatory to resolve the discrepancy. For aneurysms between 3 and $5 \mathrm{~mm}$ in maximum diameter, the results for the combination strategies were good, in particular for the CTA and TCD combination. For aneurysms less than $3 \mathrm{~mm}$ in size the sensitivity was very poor for all strategies (table 5).

Questionnaires were returned by 88\% (100/ 114) of subjects. The mean discomfort scores recorded on the visual analogue scale (with $95 \%$ CI) were: for IADSA 4.5 (3.9-5.1); for CTA 1.6 (1.2-2.0); for MRA 2.9 (2.3-3.5), and for TCD $0.5(0.3-0.7)$. Nine patients expressed no preference between any of the non-invasive modalities, four patients ranked CTA and TCD equally, and three MRA and TCD equally. Twenty four preferred CTA, 12 MRA, and 48 TCD. Trancranial Doppler was preferred to both the other non-invasive modalities by significantly more subjects $\left(p<0.001\left(\chi^{2}\right.\right.$ 39.76)) and the preference for CTA over MRA was also significant $\left(\mathrm{p}<0.025\left(\chi^{2} 7.88\right)\right)$.

\section{Discussion}

In the context of aneurysm detection by non-invasive imaging, the most important performance criterion is sensitivity. This is because there is a confirmatory reference standard method available for the non-invasive tests-namely IADSA, which carries a relatively low risk in this group of subjects $(0.07 \%$ rate of permanent neurological deficit $\left.{ }^{4}\right)$. Missing an aneurysm (false negative) is potentially disastrous for a patient whereas an unnecessary angiogram resulting from a false positive study is likely to be of much less consequence for the patient. Moreover, because it is likely that IADSA will be performed after a non-invasive test has shown a positive finding, the result on a per patient basis is more crucial for the noninvasive modalities than that on a per aneurysm basis. For example, not detecting one out of three aneurysms with a non-invasive test (true positive per patient and for two aneurysms but false negative for one aneurysm) is a much less 
important mistake than missing all the aneu$\operatorname{rysm}(\mathrm{s})$ in a particular patient (FN per patient) because in the first scenario the reference angiographic standard would be subsequently performed anyway, whereas in the second scenario it would not.

The results presented are based on the "better" of the two observers. This observer (B) had lower sensitivity but greater specificity and slightly higher accuracy than observer A (see second paragraph of results). However, the results of the two observers were very similar with good interobserver agreement $(0.69$ for CTA, 0.73 for MRA). In fact, as can be seen in the footnote to table 1 that combination strategies using the "poorer" observer's results had a better sensitivity than those for the "better" observer. Therefore using the most accurate observer's results has not biased the sensitivity results upwards at all, rather the reverse.

As outlined in the methods section, for various reasons not all patients underwent all three non-invasive imaging tests. However, the final 114 subjects included had a similar age/sex distribution and aneurysm prevalence as the original 200 subjects recruited. All the noninvasive tests were well tolerated with TCD causing the least discomfort and being preferred to the other modalities. Interestingly, CTA also caused less discomfort than MRA and was preferred by significantly more subjects despite the fact that CTA involved an injection of contrast and MRA did not (in the imaging protocol used for this study).

There are no other previous similar studies to compare with this one, where it has been possible to determine the effects of combining non-invasive tests. Combining power TCD with either CTA or MRA can produce a non-invasive imaging strategy that, compared with any single test, has improved sensitivity and level of agreement with the reference angiographic standard (on a per patient basis). This is at the cost of some subjects falling into an "uncertain" category in whom confirmatory IADSA would be required. The combination of CTA and power TCD had the greatest sensitivity (0.83) and produced the highest level of agreement with IADSA ( $\kappa 0.84)$. None the less this strategy would have led to $20 / 114$ (18\%) of subjects being classified as "uncertain" and requiring IADSA. Furthermore in the true positive and false positive cases, IADSA would also have been necessary (29 subjects and one subject respectively). Thus in the population we studied, to achieve optimal sensitivity on a per patient basis with a combination of CTA and power TCD, almost half the subjects would have undergone IADSA (50/114, 44\%).

The combination of MRA and CTA did not improve sensitivity over either examination alone, because they tended to detect or miss the same aneurysms. By detecting or missing different aneurysms, TCD did seem to complement CTA and MRA. Figure 1 indicates an example of this difference between modalities in practice. The CT angiogram in this 44 year old woman presenting with subarachnoid haemorrhage was correctly interpreted by both observers as demonstrating a $4 \mathrm{~mm}$ anterior communicating artery aneurysm (ruptured according to blood distribution on earlier plain CT) and a $3 \mathrm{~mm}$ right middle cerebral artery (MCA) aneurysm. The MR angiogram was erroneously interpreted as demonstrating only the right MCA aneurysm by both observers, whereas the power TCD demonstrated the 4 $\mathrm{mm}$ anterior communicating artery aneurysm but the ultrasonographer did not detect the right MCA aneurysm. The quality of the noninvasive imaging in this example, particularly the MRA, is limited, but this reflects the difficulty in obtaining good quality imaging in sick, anxious, and often restless patients, and the fact that in routine clinical practice not all examinations can be performed on state of the art equipment using the very latest imaging sequences.

All three of the combination strategies had a very similar overall accuracy per patient, and all had increased accuracy over the result of any single non-invasive method, but the improvement did not reach significance. However, continuing technical improvements in all the noninvasive technologies studied mean that we can expect the diagnostic performance to improve further in the near future. Combining tests together did not improve performance on a per aneurysm basis probably because in many instances the modalities disagreed on the exact location and size of an aneurysm as well as disagreeing about the presence or absence of an aneurysm in other cases. This is indicated by the many aneurysms falling into the "uncertain" category in table 3 .

It is relevant to comment that we did not use contrast enhancement for either MRA or TCD studies. This study deliberately sought to examine the effect of combining modalities that used the standard clinical imaging protocols available to most neuroscience centres. After the research started evidence began to become available that suggested contrast enhancement might improve the diagnostic performance of both MRA and TCD. However, as the evidence was early, limited, and not yet established as standard clinical practice, we decided not to alter our examination protocols part way through the study, which would not have been sound scientific practice anyway.

There is considerable data now available on the accuracy of contrast enhanced (CE) MRA in the investigation of cervical carotid, thoracoabdominal, and peripheral vasculature. ${ }^{18}$ There is evidence from in vitro models that $\mathrm{CE}$ contrast enhanced MRA improves aneurysm detection. ${ }^{19}$ However, the evidence in vivo for aneurysm detection is still very limited. It should also be remembered that the technical complexities of obtaining a good quality contrast enhanced MRA study are considerably greater than for conventional 3D TOF MRA. The timing of data acquisition and $\mathrm{K}$ space filling needs to be precisely timed to the contrast bolus to gain the benefits of improved signal to noise ration and reduced signal from adjacent venous structures. Metens et al recently published the only prospective, blinded study of aneurysm detection by 

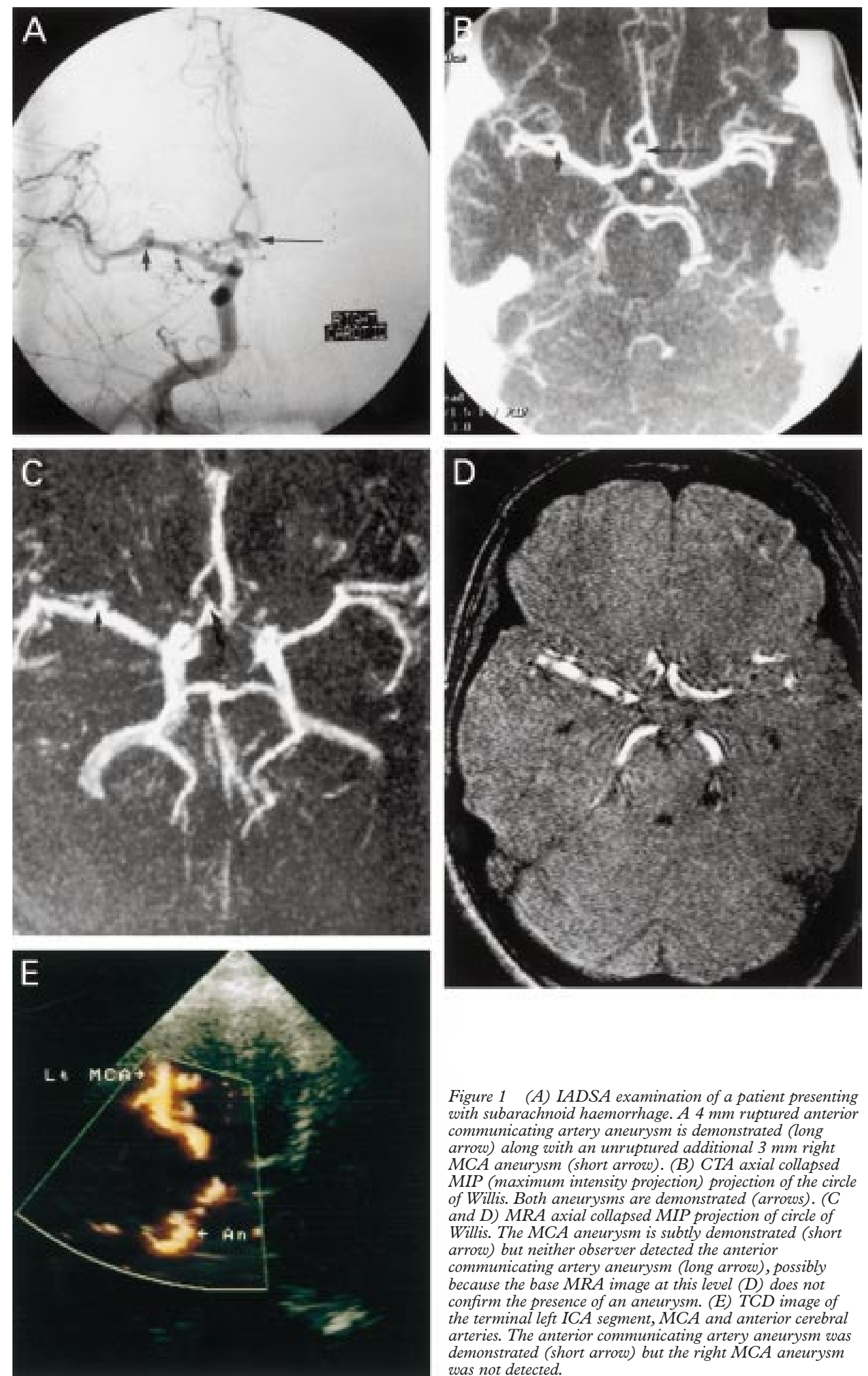

Figure 1 (A) IADSA examination of a patient presenting with subarachnoid haemorrhage. A $4 \mathrm{~mm}$ ruptured anterior communicating artery aneurysm is demonstrated (long arrow) along with an unruptured additional $3 \mathrm{~mm}$ right MCA aneurysm (short arrow). (B) CTA axial collapsed MIP (maximum intensity projection) projection of the circle of Willis. Both aneurysms are demonstrated (arrows). (C and D) MRA axial collapsed MIP projection of circle of Willis. The MCA aneurysm is subtly demonstrated (short arrow) but neither observer detected the anterior communicating artery aneurysm (long arrow), possibly because the base MRA image at this level (D) does not confirm the presence of an aneurysm. (E) TCD image of the terminal left ICA segment, MCA and anterior cerebral arteries. The anterior communicating artery aneurysm was demonstrated (short arrow) but the right MCA aneurysm was not detected.

contrast enhanced MRA to date. ${ }^{20}$ They examined 32 patients and found that contrast enhanced MRA had an improved sensitivity of $100 \%$ compared with $96 \%$ for 3 D TOF MRA but a lower specificity-94\% versus $100 \%$. The results in this small series are very impressive although this may be due in part to using a consensus result of MRA review and to the

aneurysm size - mean aneurysm size was $6 \mathrm{~mm}$ and only $39 \%(9 / 23)$ of aneurysms were $5 \mathrm{~mm}$ or less compared with $72 \%(59 / 82)$ in our study. Nevertheless detection of all small aneurysms (compared with $31 \%$ for MRA in our study) does indicate the potential improvement that contrast enhanced MRA may offer. Recent evidence has also become available to indicate 
that contrast enhancement also improves the sensitivity of power TCD. ${ }^{11}{ }^{21}$ One large series found that contrast enhancement significantly improved sensitivity from $40 \%$ to $55 \%$, although again specificity was reduced-from $91 \%$ to $83 \% .{ }^{11}$ Another much smaller series combined 3D power TCD with contrast enhancement although the ultrasonographer was not fully blinded. Klotzsch et al found that sensitivity was $87 \%$ with $100 \%$ specificity. ${ }^{21}$ In summary, both contrast enhanced MRA and power TCD look promising on the limited data available so far. It should be borne in mind, however, that the cost, invasiveness, and complexity of the examinations are increased by the use of contrast.

Increasingly there is a desire for screening tests to be carried out in general hospitals. The procedures in this study (and virtually all of those referred to in the literature) were carried out under the direct supervision of a specialist neuroradiologist in a neuroscience centre. It is doubtful whether better or even equivalent results could be achieved if the examinations were performed outside a specialist neuroimaging department. It is our view on the present evidence that these non-invasive procedures are best performed and at the very least interpreted in specialist neuroradiology departments.

Although the ISUIA data on risk of aneurysmal rupture and surgical morbidity require to be confirmed by prospective data, they provide a strong indication that most incidental aneurysms should not be considered for treatment. ${ }^{22}$ In a young asymptomatic patient with no history of subarachnoid haemorrhage, only those aneurysms larger than $10 \mathrm{~mm}$ would be regarded as possibly requiring treatment; for aneurysms smaller than this, the risks of treatment - with an overall combined surgical morbidity and mortality rate of $15.8 \%$ - seem to outweigh the risk of rupture $(0.05 \%$ per year). ${ }^{22}$ For older patients, the risks of treatment probably outweigh the rupture risk whatever the size of an incidental asymptomatic aneurysm. Table 5 indicates the performance of the non-invasive tests on a per patient basis stratified by maximum size of aneurysm. In practice, failing to detect small aneurysms in patients with no history of subarachnoid haemorrhage may not matter very much due to their very low risk of rupture, but whether asymptomatic patients should undergo any "screening" examination for intracranial aneurysms at all is highly questionable in view of the ISUIA data. Furthermore, there is a need for counselling any person considering screening regarding the diagnostic performance of available tests, the potential implications of a positive result (for example, on insurance, driving, and employment), as well as the management options available.

In conclusion, the combination of transcranial power Doppler ultrasound with either CT angiography or MR angiography did improve the detection of intracranial aneurysms compared with either modality alone on a per patient basis. Non-invasive tests even in combination cannot yet replace IADSA in the detection of small and very small aneurysms. Non-invasive imaging tests performed and interpreted in neuroscience centres are a reliable method of detecting or excluding aneurysms greater than $5 \mathrm{~mm}$ in diameter on a per patient basis - particularly when used in combination (table 5). Compared with the use of a single modality alone, employing a strategy combining two non-invasive tests would decrease the number of false negative results but at the cost of increasing the number of confirmatory IADSA studies required where the non-invasive tests do not agree. More information is still required on the diagnostic performance of CE MRA in the detection of small intracranial aneurysms.

PMW and VE were funded by the British Brain and Spine Foundation from the Davie Cooper Scottish Aneurysm Study grant, administered by the University of Glasgow. JMW was funded by the Medical Research Council under the Clinical Research Initiative in Clinical Neuroscience.

1 McFadzean RM, Teasdale EM. Computerized tomography angiography in isolated third nerve palsies. $\mathcal{f}$ Neurosurg 1998;88:679-84.

2 Velthuis BK, Rinkel GE, Ramos LP, et al. Subarachnoid hemorrhage: aneurysm detection and preoperative evaluaion with CT angiography. Radiology 1998;208:423-30

3 Young N, Dorsch NW, Kingston RJ, et al. Spiral CT scanning in the detection and evaluation of aneurysms of the circle of Willis. Surg Neurol 1998;50:50-60.

4 Cloft HJ, Joseph GJ, Dion JE. Meta-analysis of risks of cerebral angiography in patients with subarachnoid hemorhage, intracranial aneurysm and arteriovenous malformation. Stroke 1999;30:317-20.

5 Wardlaw JM, White PM. The detection and management of unruptured intracranial aneurysms. Brain 2000;123:205-

6 White PM, Wardlaw JM, Easton V. Can non-invasive imaging tests accurately detect intracranial aneurysms? A systematic review. Radiology 2000;217:361-70.

7 Ogawa T, Okudera T, Noguchi K, et al. Cerebral aneurysms: evaluation with three-dimensional CT angiography. Am $\mathfrak{f}$ Neuroradiol 1996;17:447-54.

8 Preda L, Gaetani P, Rodriguez, et al. Spiral CT angiography Preda L, Gaetani P, Rodriguez, et al. Spiral CT angiography
and surgical correlations in the evaluation of intracranial aneurysms. Eur Radiol 1998;8:739-45.

9 Huston III J, Nichols D, Luetmer P, et al. Blinded prospective evaluation of sensitivity of MR angiography to known intracranial aneurysms: importance of aneurysm size. $A m \mathcal{F}$ Neuroradiol 1994;15:1607-14

10 Aprile I. Evaluation of cerebral aneurysms with MRangiography. Rivista di Neuroradiologia 1996;9:541-50.

11 Turner CL, Kirkpatrick PJ. Detection of intracranial aneurysms with unenhanced and echo contrast enhanced transcranial power Doppler. $\mathcal{F}$ Neurol Neurosurg Psychiatry 2000;68:489-95.

12 Aaslid R, Markwalder T-M, Mornes H. Non-invasive transcranial Doppler ultrasound recording of flow velocity in basal cerebral arteries. $\mathcal{F}$ Neurosurg 1982;57:769.

13 Becker, G, Perez, J, Krone, A, et al. Transcranial color-coded real-time sonography in the evaluation of intracranial neoplasms and arteriovenous malformations. Neurosurgery 1992;31:420-8

14 Griewing B, Motsch L, Piek J, et al. Transcranial power mode Doppler duplex sonography of intracranial aneurysms. F Neuroimaging 1998;8:155-8.

15 Wardlaw JM, Cannon JC. Color transcranial "power" Doppler ultrasound of intracranial aneurysms. I Neurosurg 1996;84:459-61.

6 Armitage P, Berry G. Statistical methods in medical research. Oxford: Blackwell, 1987:117-19.

17 Altman D. Practical statistics for medical research. London: Chapman and Hall, 1991:402.

18 Prince MR, Grist TM, Debatin JF. 3D Contrast MR angiography. New York: Springer-Verlag, 1997.

19 Isoda H, Takehara Y, Isogai S, et al. MRA of intracranial aneurysm models: a comparison of contrast-enhanced three-dimensional MRA with time-of-flight MRA. $\mathcal{F}$ Comput Assist Tomogr 2000;24:308-25.

20 Metens T, Rio F, Baleriaux D, et al. Intracranial aneurysms: detection with gadolinium-enhanced dynamic threedetection with gadolinium-enhanced dynamic three-
dimensional MR angiography- initial results. Radiology dimensional MR

21 Klotzsch C, Bozzato A, Lammers G, et al. 3D-transcranial color-coded sonography of intracranial aneurysms [abstract]. Cerebrovasc Dis 1999;9(suppl 1):79.

22 International Study of Unruptured Intracranial Aneurysms (ISUIA) Investigators. Unruptured intracranial aneurysms-risk of rupture and risks of surgical intervention. N Engl f Med 1998;339:1725-33. 